\title{
Breadth, phenotype and functionality of Gag-specific $T$ cell responses induced by a heterologous DNA/MVA prime-boost HIV-1 vaccine regimen
}

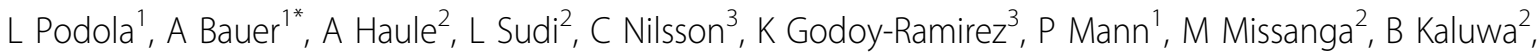

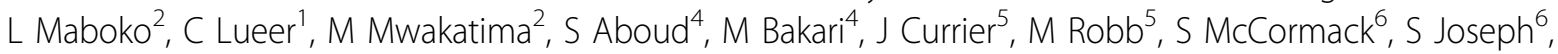 \\ E Lyamuya ${ }^{4}$, M Hoelscher $^{1}$, B Wahren ${ }^{3}$, E Sandström³ ${ }^{3}$, G Biberfeld ${ }^{3}$, C Geldmacher $^{1}$, A Kroidl ${ }^{1}$
}

From AIDS Vaccine 2012

Boston, MA, USA. 9-12 September 2012

\section{Background}

Broad Gag recognition and polyfunctionality of vaccineinduced AIDS virus-specific $\mathrm{T}$ cell responses correlate with better viral control in non-human primates and also in chronically HIV-infected individuals.

\section{Methods}

Breadth and polyfunctionality of HIV-vaccine induced Gag-specific T cell responses were investigated in healthy Tanzanian volunteers who participated in the Tanzania Mozambique HIV vaccine trial (TaMoVac 01). Vaccine recipients received $3 \mathrm{x} 0.6$ or $1.0 \mathrm{mg}$ intradermal injections of multiclade, multigene HIV-DNA vaccine boosted with $2 x$ heterologous Modified Vaccinia Ankara (MVA)CMDR. Using fresh peripheral blood mononuclear cells, the breadth of response was determined after the first MVA-CMDR boost using peptide pools for 9 successive Gag regions with an IFN-gamma ELISpot assay. Functionality (IFN-gamma, IL-2, TNF-alpha, Mip-1beta and the degranulation marker CD107) and phenotype (CD3, CD4, CD8) of HIV-specific T cells were assessed using flow cytometry in 52 participants after stimulation with peptide pools covering whole Gag-CMDR protein.

\section{Results}

Two weeks after the first MVA-CMDR boost, a median of 2 Gag regions were recognized (range: 0-9, Placebos not excluded) by 45 participants. There was a strong linear correlation between the magnitude and the breadth of

${ }^{1}$ Ludwig-Maximilians University of Munich, Mbeya, Tanzania, United Republic of Full list of author information is available at the end of the article vaccine-induced Gag recognition $(\mathrm{p}<0.0001, \mathrm{r} 2=0.44) .13$ (25\%), 13 (25\%), 3 (6\%) and 29 (56\%) of 52 subjects mounted IFN-gamma+ Gag-specific T cells that were either only CD4+, CD4+ \& CD8+, only CD8+, or CD4+ and/or CD8+, respectively. Fifty percent of IFN-gamma+ Gag-specific CD4 T cells co-expressed TNF-alpha and/or IL-2. Co-expression of Mip-1beta or CD107 was reduced compared to CMVpp65-specific CD4 T-cells, which were measured simultaneously. More than $50 \%$ of IFN-gamma+ Gag-specific CD8 T-cells co-expressed CCR5 ligand Mip1beta and a large proportion of these had degranulated.

\section{Conclusion}

The TaMoVac-01 HIV-1 vaccine regimen induces a relatively broad Gag-specific response frequently dominated by CD4 T cells, many of which co-express IL-2 and/or TNF-alpha, but also induces detectable Gag-specific CD8 $\mathrm{T}$-cells in a third of vaccine recipients.

\section{Author details}

'Ludwig-Maximilians University of Munich, Mbeya, Tanzania, United Republic of ${ }^{2}$ Mbeya Medical Research Programme, Mbeya, Tanzania, United Republic of. ${ }^{3}$ Karolinska Institute and Swedish Institute for Communicable Disease, Solna, Sweden. ${ }^{4}$ Muhimbili University of Health and Allied Sciences (MUHAS), Dar Es Salaam, Tanzania, United Republic of. ${ }^{5}$ US Military HIV Research Program, Rockville, MD, USA. ${ }^{6}$ MRC Clinical Trials Unit, London, UK.

Published: 13 September 2012

doi:10.1186/1742-4690-9-S2-P273

Cite this article as: Podola et al:: Breadth, phenotype and functionality of Gag-specific T cell responses induced by a heterologous DNA/MVA prime-boost HIV-1 vaccine regimen. Retrovirology 2012 9(Suppl 2):P273. 